\title{
Study on the Wireless Heat Meters
}

\author{
Rujian Ma, Xueyan Yang, Xiao Wang, Enping Zhang \\ School of Control Science and Engineering, University of Jinan, Jinan, China \\ E-mail:rjma@ujn.edu.cn \\ Received August 16, 2009; revised September 21, 2009; accepted October 19, 2009
}

\begin{abstract}
The design of a new type heat meters based on RFID technology are presented in this paper. By use of RFID technology in heat meters, the data can be exchanged between heat meters and heat supplying department by RF cards. The information can be transmitted in a non-contact way. In this way, the purpose of automatic identification can be achieved. The experimental study of the heat meters is also performed in the paper. The results show that the new type of heat meters can meet the demands of users. Compared with the ordinary heat meters, the new type of heat meters have the advantages of small in meter volume, high accuracy, no impact of water quality and good reliability.
\end{abstract}

Keywords: Heat Meters, Design, Experimental Study, RFID

\section{Introduction}

Radio frequency identification (RFID) technology is a non-contact automatic identification technology [1]. Based on the wireless data communication technology, the RFID technology has been widely used for wireless remote meter-reading technology and provides a sufficient technology foundation. At the present, the central heating supply in the urban area of China, waste of energy and unreasonable charges by the heating area, these problems have restricted the further development of central heating. In the implementation of structural reform in the urban heating, heat meters are the key equipment in heating metering and charging control system, which can achieve the metering of urban central heating household and energy charges in accordance with the implementation of heat, and this has gradually become a new hot industry. To make the study of intelligent heat meters based on RFID technology, we consulted the International Organization of Legal Metrology OIML-R75 rules and the European standards EN1434 heat meters which are most advanced in the world, also we draw lessons from European advanced technology and experience of similar products, these heat meters can realize not only for the centralized hot household heat metering, but also the non-contact measurement of data transmission and the control of clearing transactions. As a result, the heat meters provide the possibility of intelligent management in cities and a firm material foundation for the digital communities and cities, which can bring high social and economic benefits.
Heat meters are the terminal equipments of the heat metering management system, as shown in Figure 1. They exchange data with the department of heat metering by radio frequency (RF) cards. As an important aspect of RFID technology, the RF cards achieve noncontact transmission of information by using radio frequency signals through space coupling. Furthermore, they can achieve the purpose of automatic identification through the transmission of information.

In heat metering management system, each user gets a new RF card when installed new equipment. Before using heat meters, users must open accounts in the department and the management system establishes detailed information for each user. After opening accounts, users buy heat according to their actual needs of the heat required and bring about closer ties between RF card and their own heat meters. From the information security point of view, in the card all user information, heat data calorie and other management information is transmitted

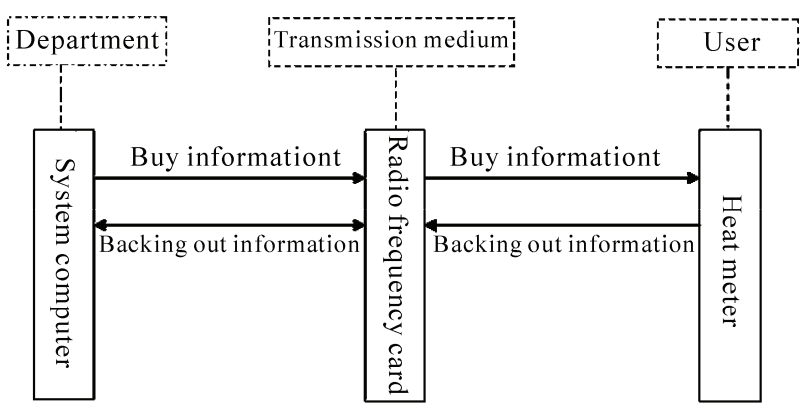

Figure 1. Diagram of the heat supplying management system. 
in the form of encryption, heat meters do the corresponding decryption work when they accept. At the same time, heat meters transmit the user's use of heat, the remaining heat value, the corresponding record in history and heat meters state information to heat supplying department by RF card. Heat supplying department analyses and compares the historical data they collected, and obtains a large number of heating data, these data provide strong data support for the department to make decisions.

\section{Design of Heat Meters}

Heat meters consist of two major parts, the RF card and smart heat metes.

The Mifare One of Philips chips (referred to M1 card) is a kind of commonly used RF card. The parameters of the card are: $8 \mathrm{~K}$ in storage capacity, 10 years of data retention, 100,000 cycles for rewriting, unlimited time for reading, and no battery needed. The card has its own antenna, encryption control logic circuit and communication logic circuit, inter-national common DES and cross-RES secrecy algorithm is adopted in the communication between the card and reader, its confidentiality performance is very high, with characteristics of fast anti-collision, high reliability in data communication, anti-strong electrical interference, moisture-proof, waterproof, convenience, fast and so on [2].

The smart heat metes contains a microprocessor, a mechanical rotor-type non-magnetic flow sensor, a pair of temperature sensor, a voltage monitor and radio frequency modules, and so on. The overall structure of the heat meters is shown in Figure 2.

The microprocessor uses singlechip of MSP430FW425 type for the wireless long-distance transmission calorimeter. Its internal composite signal processor is key microprocessor of integrating monitor, superior performance, reliable and cost-effective [3]. The current sensor is mechanical rotor type multi-striation flowmeter which is improved to comply with the request of non-magnetic current signal acquisition calibration module and measurement characteristic. It is characterized by a small wheel rotor installed. Signal damping board is light in weight, wide flow measurement, and high accuracy of flow measurement and applicable to poor quality

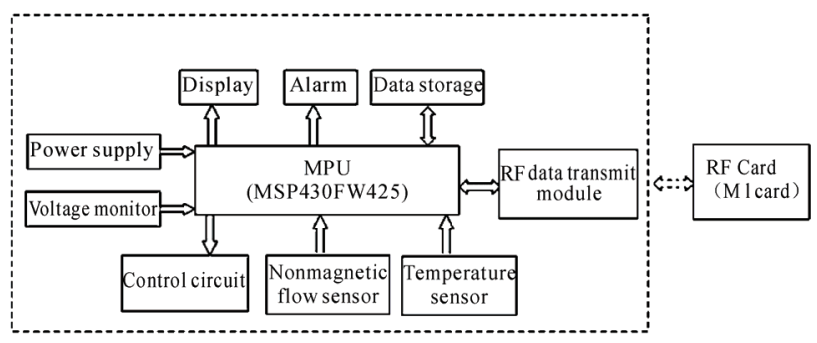

Figure 2. Overall structure diagram of heat meters. water etc. [4]. A pair of temperature sensors are used for measuring temperature and signal of difference in temperature about heat exchanger inlet and outlet, the precision of the sensors less than $0.1^{\circ} \mathrm{C}$ [5]. Integrating monitor is hardcore. On one hand, it collects and calibrates the flow rate signal, temperature and signal of difference in temperature integration demonstration quantity of heat of non-magnetic flowmeter. On the other hand, it uses RF transport module to transports data.

Control software of heat meters are designed based on the modularizaion method, including radio frequency read-write module, non-magnetic flowmeter scan module, flow rate module and so on. Each part functionally independent designed, effectively to guarantee the reliability of the scale of intelligent hot work. The table with applicability and flexibility and humanized design concept are full considered in the design, heat meters based on RFID technology has superior intelligence and fully meets the market demand. Flow chart of the main program and the card interrupt program are shown in Figures 3 and 4.

\section{Experimental Study}

Three heat meters of DN20 type are used for the prototype test. The test includes accelerated wear test, metrological characteristic test and data communication test.

The accelerated wear test requires that the meter is continuously running 300 hours under the maximum flow rates. The maximum flow rate of this meter is 5000 $\mathrm{kg} / \mathrm{h}$. All the three meters are in good working situation after the test.

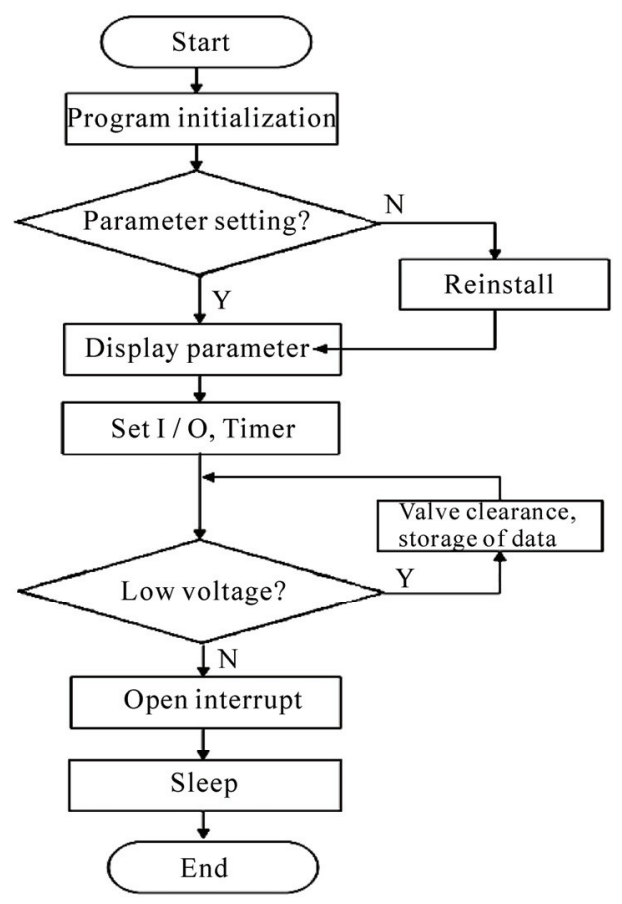

Figure 3. Flow chart of the software program. 


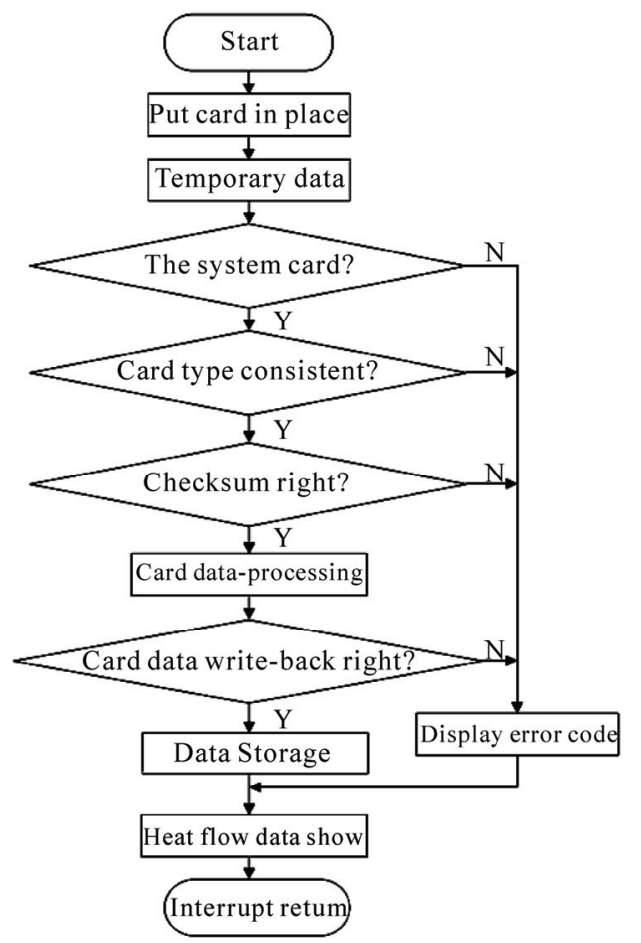

Figure 4. Flow chart of the card interrupt program.

Metrological characteristic test includes temperature test and flow rate test. The measuring points for the temperature test are 10, 50 and 90 degrees Centigrade, representing the low, medium and high temperatures respectively. The measurement results of temperatures are listed in Table 1. It can be seen from the table that all the data is less than the permit error of level 2 meters $(3.5 \%)$. The measurement of flow rates is performed in three temperature points representing the low, medium and high temperatures. The results are shown in Figures 5, 6 and 7. It can be seen from the figures that all the data is less than the permit error of level 2 meters.

The test items of data communication test include Distance of data reading \& writing, resetting data, data summation, data transfer and replacement of cards and meters. The measurement results of the data communication

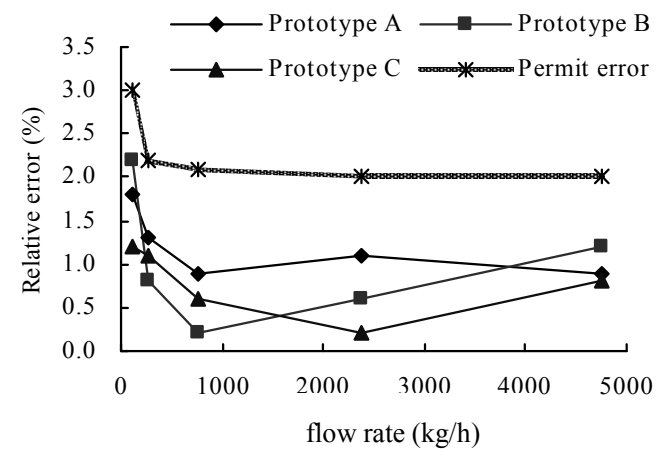

Figure 5. Measurement errors of flow rates at 10 degree Centigrade.

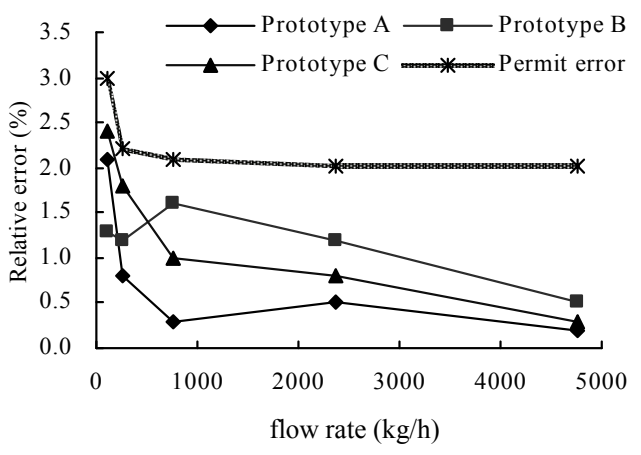

Figure 6. Measurement errors of flow rates at 50 degree Centigrade.

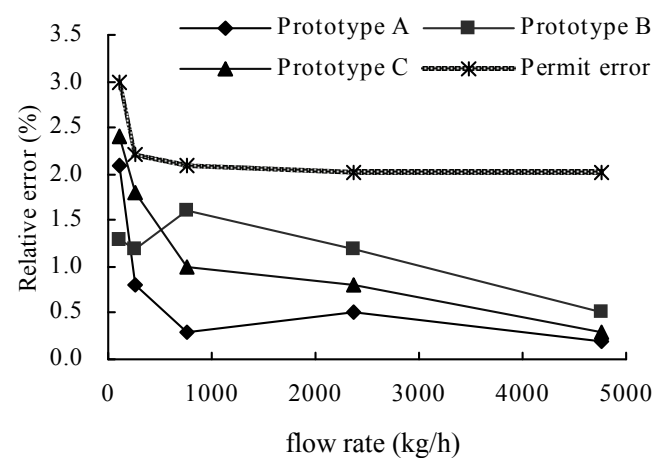

Figure 7. Measurement errors of flow rates at 90 degree Centigrade.

test are listed in Table 2. Compared with the performance required of the meters (also listed in the table) indicates that all the performance indexes are satisfied with the requirement of the meters.

Table 1. Measurement results of temperatures.

\begin{tabular}{cccc}
\hline Temperature & \multicolumn{3}{c}{ Maximum errors (\%) } \\
\cline { 2 - 4 } Test points $\left({ }^{\circ} \mathrm{C}\right)$ & Prototype A & Prototype B & Prototype C \\
\hline 10,50 and 90 & 1.17 & 1.92 & 0.79 \\
\hline
\end{tabular}

Table 2. Results of data communication tests.

\begin{tabular}{lllll}
\hline \multirow{2}{*}{ Test items } & \multicolumn{1}{c}{$\begin{array}{c}\text { Performance } \\
\text { required }\end{array}$} & \multicolumn{3}{c}{ Prototype numbers } \\
\cline { 5 - 5 } $\begin{array}{l}\text { Distance of } \\
\text { data reading } \\
\text { \& writing }\end{array}$ & $\geq 2.0 \mathrm{~cm}$ & No. B & No. C \\
$\begin{array}{lllll}\text { Resetting } \\
\text { data }\end{array}$ & $\begin{array}{l}\text { The valve is } \\
\text { closed after data } \\
\text { resetting }\end{array}$ & Normal & Normal & Normal \\
& $\begin{array}{l}\text { The valve will } \\
\text { be open after } \\
\text { summation }\end{array}$ & Normal & Normal & Normal \\
& $\begin{array}{l}\text { data summation } \\
\text { Will be work }\end{array}$ & & & \\
$\begin{array}{l}\text { Data } \\
\text { transfer }\end{array}$ & $\begin{array}{l}\text { normally after } \\
\text { the data transfer }\end{array}$ & Normal & Normal & Normal \\
$\begin{array}{l}\text { Replacement } \\
\text { of cards and } \\
\text { meters }\end{array}$ & $\begin{array}{l}\text { Will be work } \\
\text { normally after } \\
\text { the replacement }\end{array}$ & Normal & Normal & Normal \\
\hline
\end{tabular}




\section{Conclusions}

A new type of heat meter is designed based on RFID technology in this paper. This type of heat meters can provide centralized heat metering, residence intelligence, management digitization and information system management with new plan. The experimental study is also presented in the paper. The results indicate that the heat meters have high accuracy in measurement, no impact of water quality, good reliability and low power consumption.

\section{Acknowledgements}

This project is supported by Research Foundation of University of Jinan (XKY0806) and Key Subject Research Foundation of Shandong Province.

\section{References}

[1] Y. Shen, "Application of RFID technology in management of college teaching," Instrumentation Analysis Monitoring, No. 1, 2008.

[2] X. Yi, "Application of Mifare one RF card in prepayment electric meter," Process Automation Instrumentation, Vol. 28, No. 8, 2007.

[3] S. Guo, "Design of RFID reader based on MCUMSP430," Journal of Jilin University, Vol. 28, No. 2, 2007.

[4] X. Yang, "Design of digital water meter based on MSP430FW425 MCU scan IF module," Computer Engineering and Applications, Vol. 42, No. 18,42, 2006.

[5] X. Wei, "MSP430 singlechip interface technology and system design examples," Beijing University of Aeronautics \& Astronautics Press, 2002. 